Besondere Beachtung verdienen die Arbeiten von Rosenmund und Zetsche itber die Beeinflussung der Katalysatoren durch gewisse Zusätze, wodurch erreicht werden kann, dass die Katalyse nicht bis zu dem theoretisch zu erwartenden Ende fortschreitet, sondern bei einer gewiunschten $\mathrm{Zwischenstufe} \mathrm{halt.} \mathrm{Allerdings}$ hat sich die Untersuchung der Forscher auf die Fetthartung noch nicht ausgedehnt und doch wäre es von Wert, wenn man z. B. die Linolsäure oder die Linolensäure nur bis zur Oelsăure hydrieren könnte. Auch die Stellung der Doppelbindungen in der Kohlenstoffkette der Fettsäuren spielt eine wichtige Rolle. Nach Moore lassen sich alle übrigen Doppelbindungen leichter absättigen als die 9,10-Stellung; dass bei der Härtung Oelsäuren (Isoölsăure, Elaidinsulure usw.) entstehen, die in dem ursprünglichen Oel nicht vorhanden waren, wird daraut zurtickgetührt, dass bei Anwesenheit mehrerer Doppelbindungen diese nicht der Reihe nach gesättigt werden, sonderu dass die Doppelbindung zuerst angegriffen wird, die zutällig rein mechanisch zugleich mit Wasserstoff und einem Katalysatorteilchen in Berührung kommt. Die Bildung verschiedener Isomeren dürtte viellejcht aber anch auf eine Rückläufigkeit der Katalyse zurïckzuführen sein, die unter gewissen Bedingungen eintreten kann. Le vey behandelt Oel mit Nickelkatalysator in einem Gasstrom bei $220-250^{\circ}$, wobei eine Abspaltung von Wasserstoft erfolgt, und aus nichttrocknenden Oelen unter Erhöhung der Jodzahl solche mit Trockeneigenschaften entstehen. Verschiedene Gase sind branchbar, sogar Wasserstoff, jedoch ist Kohlenoxyd das günstigte. Es ist dies eine Beobachtung, welche anch der Vortragende selber gemacht hat, nämlich dass gegen das Ende der Härtung die Jodzahlen wieder zunehmen, statt auf Null herunterzugehen.

Levey fand dann auch, dass ein Oel mit hoher Jodzahl, aber schlechter Trockeneigenschaft, in ein gut trocknendes Oel libergefiulurt werden kann, wenn es erst teilweise mit Wasserstoff gesättigt, und darauf nach seinem Vertahren wieder Wasserstoff abspaltet.

Der Gedanke, die Wasserstoffanlagerung nicht, wie Sabatier es tat, im Gas- und Damprastande, sondern im flüssigen Zustande des zu süttigeuden Stoffes vorzunehmen, hat sich in der Fetthartung als erfolgreich erwiesen und ist weit über diese hinaus angevandt worden (z. B. Tetralinfabrikation).

Die Wirtschaftliche Seite der Fetthärtung bildet der dritte Teil des Vortrages.

Die Fethärtung hat sich in einem Zeitraum von etwa 15 Jahren als eine eigene selbstăndige Industrie tuber die ganze Erde verbreitet. Die erste fabrik. massige Aulage war bei der Firma Crosfield in England. Im Jahr 1914 sollen im ganzen 24 Härtungs- fabriken bestanden baben, davon 18 in Europa und von diesen 6 in Deutschland, mit einer Jahreserzengung von 200-300 Millionen kg Fett. Jetzt sind es, einschliesslich Deutschland mit ungefahr 11, etwa 60 Fabriken wozu noch 14-15 Fabriken in U.S. Amerika kommen, mit einer angeblichen jährlichen Leistungsfähigkeit voll $142000 \mathrm{t}$, die aber in den letzten Jahren tatsüchlich nur etwa halb so gross war.

Als Rohstoffe kommen für die Härtung sämtliche Oele in Betracht, die in technischem Masse gewonnen worden. Verwendung finden die gehärteten Fette für die Seifentabrikation und für die menschliche Ernährung. Bei uns wandern sie fast durchweg in die Margarinefabriken; in Amerika sind sie auch als Schmalzersatz sehr beliebt.

Besonders während des Krieges spielte die Fettliartung für die deutsche Volksernăhrnng eine bedeutende Rolle. Da durch die Härtung der eigentümliche unangenehme Geruch und Geschmack mancher Oele vollständig beseitigt wird, so dass auch solche Oele in brauchbare Speisefette übergefübrt werden konnten. Gebärteter Tran als Speisefett ist aber nicht nur als eine Kriegserscheinung anzusehen, sondern er ist imstande, eine tadellose Margarine zu ergeben, wodurch der Walfischtang so grossen Aufschwung nahm, dass im Norden dieses Seewild fast ausgerotter ist, und die Fethärtung immer mehr auf die Pflanzenöle angewiesen wird.

Ueber die Erzeugung an gehärteten Oelen und über die Fettmengen, welche die Margarinefabriken in den Jahren 1915 bis 1919 vom Kriegsausschuss zugewiesen wurden sind berichtet der Vortragende an Hand von Tabellen. So betrug die Menge von gehärtetem Fett für letzteren Zweck i. J. 191612978 t, i. J. 19177714 t, i. J. 191815247 und i. J. 191930421 t.

Wenn die Fetthärtung in den ersten Jaliren nach ihrer Erfindung nicht sotort festen Fuss fassen konnte, so war darau die schlechte Marktlage schnld, indem nămlich die Preise für die Oele derart hoch waren, dass nur ganz geringe Herstellungskosten berechnet werden durften, um im Preise mit den natïrlichen harten Fetten konkurrieren zu können. Eine ähnliche Marktlage herrscht anscheinend seit 1918 wieder in Amerika. Jedoch darf dieser Zustand nicht als dauernd angesehen werden, und die Marktlage hat sich immer wieder in der Weise geändert, dass sich die Härtungsindustrie mit Erfolg durchsetzen konnte. Ihren volkswirtschaftlichen Wert als machtvoller Regulator des Preisverhaltnisses zwischen flüssigen Oelen und festen Fetten wie durch die Vergrösserung des Weltvorrats an Nahrungsfetten wird die Hartungsindustrie erst dann verlieren, wenn das Gute, das sie uns gebracht bat, durch etwas Besseres abgelöst wird. (Zeitschr. f. angew. Chem.). $\mathrm{Br}$.

\title{
Die Carnaubawachs-PaIme.
}

Bearbeitet durch Fa. Lndmar m. b. H., Köln a./Rhein.

Da die Kenntnisse uber die CarnaubawachsPalme, deren vielseitige und nutzliche Verwendungsmöglichkeiten, sowie uber die Gewinnung des Carnaubawachs selbst, nicht so sehr verbreitet sind, wie es wünschenswert wäre, versuchen nachstehende Ausführungen, unter Benutzung verschiedener Quellen, diese Lticke auszufullen.

Die Carnaubawachs-Palme gehört zur
Gattung Copernicia, die man in sechs Arten einteilt; sämtliche haben ihre Heimat im tropischen Amerika. Die fur den Handel hauptsächlich in Frage kommende und infolgedessen meist bekannte Art, welche auch kurz Carnaubapalme genannt wird, hat einen sechs bis $\mathrm{z}$ wölf Meter hohen, mit Blattnarben bedeckten Stamm von $30-45 \mathrm{~cm}$. Umfang. Ihre sechs bis acht jüngsten Blätter zeichnen sich 
dadurch aus, dass sie an den gegenseitigen Berlthrungspunkten durch eine harzartige Masse zusammengehalten werden. Die tiefgespaltenen Fächerblätter bilden eine fast kugelige Krone. Die Fiedern bleiben getrennt, jedoch vereinigen sich die Blätter wieder am Stammkopf, wodurch die Kugelform entsteht. Die jungen Blätter sind an den unteren Seiten klargelb; in diesem Stadium ihrer Entwicklung sondern sie einen trockenen, pulverförmigen, aschfarbigen Stoff ab, dem ein eigentümlicher aber angenehmer Geruch anhaftet. Dieser Stoff wird in der Botanik als Pflanzenwachs bezeichnet. Er hängt so lose an den Blättern, dass er abgeschtittelt werden kann, d. h. solange die Blätter jung sind. Bei fortgeschrittener Entwicklung, wenn sie die bereits erwähnte Kugelform gebildet haben, gentigt schon ein Windhauch, um das Wachspulver zu zerstreuen. Die kleinen grüen Bliten sitzen an weitverzweigten, in den Achsen der Blätter stehenden, Blutenständen, woraus sich dann die Frlichte entwickeln.

Den gunstigsten Boden für das Gedeihen der Carnaubapalme bilden die trockensten Gegenden des tropischen Brasilien; man findet dort sehr ausgedehnte Palmenhaine. Selbst nach zwei oder dreijähriger absoluter Trockenheit, während welcher Zeit fast die ganze ubrige Vegetation der unbarmherzigen tropischen Sonnenglut erliegt, macht die Carnaubapalme eine Ausnahme, in reicher Blütenpracht und lippigem Grun beherrscht sie die Zerstörung. Sie soll so unempfindlich gegen Hitze sein, dass selbst das Feuer ihre Lebenskraft nicht vernichten könne. Andererseits ist die Palme auch wieder sehr empfindlich, denn ein Regenfall in der nassen Jahreszeit und eine nachfolgende zu lange Dürre, beein. trächtigen die Leistungsfähigkeit in solchem Masse, dass wie die Erfahrung im letzten Jahrzehnt gezeigt hat oft Fehlernten stattinden. Am besten entspricht dem Gedeihen der Palme ein trockener den Ueberschwemmungen nie ausgesetzter Standort.

Am häufigsten kommt die Carnaubapalme in der Provinz Ceara vor; ferner in den Tälern von Assu und Apody im Staate Rio Grande do Norte. Diese Palmenhaine, die sich von Ort zu Ort längs den Flussläufen hinziehen, sind die einzigen Waldungen, welche die Flussebenen in ihrer ganzen Breite bedecken und dem Beschauer ein tropischmysteriöses Landschaftsbild darbieten. Der Reiz der Landschaft wird noch ganz besonders dadurch gehoben, dass diese Wälder durchweg nur aus schlanken Palmen, ohne das sonst in tropischen Gebieten typische unentwirrbares Schlinggewächs und Unterholz, erhaben und grandios die weiten Flächen beherrschen.

Es gibt wohl in der Natur wenig andere Pflanzen, die der Nutzlichkeit der Carnaubapalme gleich kommen. Die Blattrippen liefern als Tukumfasern Besenmaterial. Ausserdem finden die Fasern vielfach Verwendung zur Herstellung von Stricken, Seilen und Fischnetzen. Die Einwohner Brasiliens fertigen Gewebe fur verschiedenste Verwendungszwecke daraus, ferner Ersatz europäischer Strohgeflechte wie Matten, Hute, Körbe, Stopfinaterial fur Matratzen und Kissen. Fur den dritten Teil der in der Provinz Ceara benötigten Seilerwaren liefert die Carnaubapalme den Rohstoff. Die Zubereitung der Fasern für industrielle $Z$ wecke ist sehr einfach. Die Blätter unterliegen keinem Røstverfahren wie andere Faserpflanzen, sondern man schneidet sie in Streifen und hechelt sie auf Brettern, die mit Nägeln und Fischzähnen besetzt sind.

Die Blätter dienen als Bedachung von Hutten, diese ist leicht, undurchdringlich und von gefälligem Aussehen. Die grünen Früchte werden von den Eingeborenen zum Teil als Viehfutter und allch trotz ihres bitteren Geschmacks als Nahrungsmittel für Menschen verwendet. Selbst die ölhaltigen Samen werden zuweilen von der weissen Bevölkerung in geröstetem Zustand als Ersatz für Kaffee genossen. Beim Anzapfen der Palme sondert der Stamm eine sirupartige Flussigkeit $a b$, die ebenfalls für Menschen genussfähig ist und sich bei den Eingeborenen als Erfrischungsgetränkt grosser Beliebtheit erfreut. Die Wurzel findet zur Herstellung verschiedener Heilmittel Verwendung. Abgesehen von dieser allgemeinen Nutzlichkeit der Carnaubapalme ist sie den Bewohnern der durch die Hitze heimgesuchten brasilianischen Gegenden geradezu unentbehrlich geworden, da sie durch ihre Verbreitung einen natürlichen Schutz gegen die alles versengende Sonnenglut gewährt. Wird die Palme im hohen Alter gefällt, so dient das sehr harte Holz zu Bauund Tischlerzwecken. Die Farbe des Holzes ist gelblichrot und von schwarzen Adern durchzogen. Das Holz hat den Vurteil, eine schöne Politur anzunehmen, von Insekten nicht angegriffen zu werden und als Schiffbaumaterial im Seewasser eine lange Lebensdauer zu gewährleisten.
(Schluss folgt.) 